\title{
Oral Dextran Sulfate Sodium Administration Induces Peripheral Spondyloarthritis Features in SKG Mice Accompanied by Intestinal Bacterial Translocation and Systemic Th1 and Th17 Cell Activation.
}

\author{
Yuya Tabuchi \\ Kyoto University \\ Masao Katsushima \\ Kyoto University \\ Yuri Nishida \\ Kyoto University \\ Mirei Shirakashi \\ Kyoto University \\ Hideaki Tsuji \\ Kyoto University
}

Hideo Onizawa

Kyoto University

Koji Kitagori

Kyoto University

Shuji Akizuki

Kyoto University

Ran Nakashima

Kyoto University

Kosaku Murakami

Kyoto University

Koichi Murata

Kyoto University

Hajime Yoshifuji

Kyoto University

Masao Tanaka

Kyoto University

Akio Morinobu

Kyoto University

Motomu Hashimoto ( $\nabla$ hashimoto.motomu@med.osaka-cu.ac.jp )

Osaka City University 


\section{Research Article}

Keywords: Spondyloarthritis (SpA), inflammatory bowel disease (IBD), dextran sulfate sodium (DSS), quantitative polymerase chain reaction (qPCR), next-generation sequencing (NGS), splenic Th1 and Th17 cell

Posted Date: November 24th, 2021

DOI: https://doi.org/10.21203/rs.3.rs-1071333/v1

License: (c) (i) This work is licensed under a Creative Commons Attribution 4.0 International License. Read Full License 


\section{Abstract}

Background: Spondyloarthritis (SpA) is an autoimmune and autoinflammatory musculoskeletal disease characterised by systemic enthesitis. Recent research has focused on subclinical inflammatory bowel disease (IBD) in SpA pathogenesis. SKG mice, harbouring the Zap70 W163C mutation, increase autoreactive Th17 cells intrinsically, and show SpA features, including enteritis, after peritoneal injection of $\beta-1,3$ - glucan under SPF conditions. In a conventional environment, they exhibit spontaneous arthritis with fungal factors. This study aimed to clarify whether oral dextran sulfate sodium (DSS) administration, utilised in IBD model mice, can provoke SpA features in SKG mice under SPF conditions, focusing on the relationship between gut microorganisms and SpA pathogenesis.

Methods: SKG and BALB/c mice were administered oral DSS, and their body weights, arthritis, and enthesitis scores were recorded. In another cohorts, antibiotics (meropenem and vancomycin) or an antifungal agent (amphotericin B) were administered orally before DSS administration. The splenic Th1 and Th17 cell populations were examined before and after DSS administration using flow cytometry. Furthermore, the amount of circulating bacterial DNA in whole blood was measured by absolute quantitative polymerase chain reaction (qPCR), and the number and characteristics of bacterial species corresponding to these circulating DNA were analised by next-generation sequencing (NGS).

Results: Ankle enthesitis as a peripheral SpA feature was elicited in half of DSS-administered SKG mice, and none of the BALB/c mice. Pre-administration of antibiotics suppressed enthesitis, whilst an antifungal agent could not. Th1 and Th17 cell levels in the spleen increased after DSS administration, and this was suppressed by pre-administration of antibiotics. SKG mice have a larger amount of bacterial DNA in whole blood than BALB/c mice before and one day after the initiation of DSS administration. The number of bacterial species in whole blood increased after DSS administration in SKG and BALB/c mice. Some genera and species significantly specific to the DSS-treated SKG mice group were also detected.

Conclusion: Oral DSS administration alone elicited peripheral enthesitis in SKG mice with bacterial translocation accompanied by increased splenic Th1 and Th17 cell levels. Pre-administration of antibiotics ameliorated these DSS-induced SpA features. These findings suggest that intestinal bacterial leakage plays a pivotal role in SpA pathogenesis.

\section{Introduction}

Spondyloarthritis $(\mathrm{SpA})$ is an autoimmune and autoinflammatory musculoskeletal (MSK) disease characterised by systemic enthesitis [1], comprising ankylosing spondylitis (AS), psoriatic arthritis (PsA), IBD-associated arthritis, and reactive arthritis (ReA) [2]. Genetic backgrounds, including HLA-B27 positivity, mechanical stress, and dysbiosis, have been investigated in the pathogenesis of SpA [3]. In addition, subclinical inflammatory bowel disease (IBD) has recently attracted attention. According to some reports, approximately half of patients with AS have subclinical microscopic IBD [4]. Another report has shown an increase in faecal calprotectin levels in patients with AS [5]. An increasing number of 
researchers are studying the relationship between MSK manifestations and gut inflammation in the pathogenesis of SpA [6]. However, there are still many unrevealed disease mechanisms .

Animal models are essential for clarifying the complicated pathogenesis of SpA. SKG mice, identified by Sakaguchi et al, possess the Zap70 W163C mutation [7]. In a conventional environment, they are known for their intrinsically increased autoreactive Th17 cells and spontaneous IL-17A-dependent autoimmune inflammatory arthritis [8]. Reports show that spontaneous arthritis in SKG mice under conventional conditions and $\beta$-glucan-induced arthritis under specific pathogen-free (SPF) conditions are related to the dectin-1 pathway. Furthermore, intraperitoneal injection of an anti-fungal agent, amphotericin B, prevents the onset of arthritis completely in a conventional environment and partially under SPF conditions [9]. It has also been shown that intraperitoneal injection of curdlan, $\beta-1,3-D$-glucan, causes MSK SpA features and IBD-like enteritis in SKG mice under SPF conditions [10]. The same group has shown that the Zap70 mutation in SKG mice disrupts the homeostatic relationship between gut microbiota and the host, and that SKG mice have intrinsic gut dysbiosis due to their genetic background [11]. They also demonstrated that intestinal IL-23 plays a pivotal role in the pathogenesis of $\beta$-glucan-induced SpA pathogenesis in SKG mice [12] . In humans, anti-Saccharomyces cerevisiae antibodies (ASCAs) are detected in Crohn's disease patients and in AS patients [13-15]. The importance of the IL-23-IL-17 axis has been emphasised also in human SpA pathogenesis [16]. However, little is known about whether oral administration of dextran sulfate sodium (DSS), which is commonly used in studies regarding IBDs, has the potential to cause SpA features, including peripheral enthesitis in SKG mice, and if so, what the mechanisms are like.

This study investigated whether oral DSS administration under SPF conditions solely provokes enthesitis as a SpA feature in SKG mice, focusing on the relationship between bacterial and/or fungal translocation and SpA pathogenesis.

\section{Methods}

\section{Mice, induction of disease and reagents}

BALB/c mice were purchased from Claire Japan, Inc. (Tokyo, Japan). SKG mice were obtained from Prof. Sakaguchi (Osaka University, Osaka, Japan). Both mice were bred under SPF conditions at the main facility of the Institute of Laboratory Animals of Kyoto University. All experiments were approved by the Kyoto University Animal Ethics Committee. In all experiments, female mice 8 to 12 weeks of age were used. In the DSS administration cohorts, 1\% DSS (MP Biomedicals, Santa Ana, CA, USA, molecular weight 35.000-50,000 kDa) in drinking water was administered for the first two consecutive weeks. In the set of cohorts with antibiotic agents, $6 \%$ dimethyl sulfoxide (DMSO) (Nacalai Tesque Inc., Tokyo, Japan) as a control group, 6\% DMSO and meropenem trihydrate (MEPM) (1 g/L) (FUJIFILM Wako Chemicals Inc., Tokyo, Japan) and vancomycin hydrochloride (VCM) (0.5 g/L) (Nacalai Tesque Inc.), and 6\% DMSO and amphotericin B (AMPH-B) ( $0.3 \mathrm{~g} / \mathrm{L}$ ) (Nacalai Tesque Inc.) were administered respectively for 1 week in drinking water, from the time point 2 weeks before the initiation of DSS administration. Ten mice were 
used per group to confirm disease phenotypes, and five mice were used per group in other repeated sets of cohorts for the FCM, QPCR, and NGS analyses.

\section{Histologic analysis}

The ankle enthesis and caudal vertebral joints from BALB/c mice and SKG mice with or without DSS treatment were fixed in formalin and embedded in paraffin at the experimental endpoints. Mice with swollen ankles were selected for DSS-treated SKG mice. Four-micrometre sections were cut and stained with hematoxylin and eosin (H\&E). Photographs were taken with an Olympus CKX31 microscope and an Olympus STYLUS 1s camera.

\section{Scoring of clinical signs}

Clinical features of the mice were scored every other week by the same observer. A conventional scoring system by Sakaguchi et al. was used to evaluate arthritis [7]. The ankle was scored on each side to evaluate enthesitis, based on our original criteria: 0.5 = deviation of plantar aponeurosis (the midline of the plantar aponeurosis indicating a toe other than the third one), $1.0=$ uplift of the plantar aponeurosis over the angle of the ankle (Fig. 1g). The scores of both ankles were summed, and the maximum possible enthesitis score was 2.0.

\section{Flow cytometry}

Single -cell suspensions were prepared by triturating the spleens between the ends of sterile frosted slides and filtration through nylon mesh. After erythrocyte lysis, splenocytes were suspended in RPMI 1640 tissue medium supplemented with $10 \%$ heat-inactivated foetal bovine serum, $50 \mathrm{U} / \mathrm{mL}$ penicillin, and $50 \mu \mathrm{g} / \mathrm{mL}$ streptomycin, and cultured for $3 \mathrm{~h}$ at $37^{\circ} \mathrm{C}$ with Cell Stimulation Cocktail (eBioscience, CA, USA). After FcyR blockade with anti-CD16/32 antibodies (BioLegend, CA, USA), extracellular antigens were stained for 20 min at $4{ }^{\circ} \mathrm{C}$ in RPMI 1640 medium. Cells were fixed and permeabilised using Foxp3/Transcription Factor Buffer Set (eBioscience) and stained for intracellular cytokines. Samples were acquired using a BD FACSCalibur flow cytometer operated by CellQuest (BD Biosciences, CA, USA), and the data were analysed using FlowJo software (BD Biosciences). Cell populations were identified by staining with anti-CD3-FITC (17A2), anti-CD4-PerCp/Cy5.5 (GK1.5), anti-IL-17A-PE (TC11-18H10), and anti-IFN-Y-Alexa Flour 647 (XMG1.2) (BioLegend).

\section{DNA extraction and quantitative polymerase chain reaction}

Whole blood $(200 \mu \mathrm{L})$ was collected in DNA-free tubes from each mouse using a Goldenrod Animal Lancet (Braintree Scientific Inc., MA, USA), quickly preserved at $4{ }^{\circ} \mathrm{C}$, and then frozen at $-30{ }^{\circ} \mathrm{C}$ until DNA extraction. NucleoSpin Blood QuickPure (MACHEREY-NAGEL GmbH \& Co. KG, Dueren, Germany) was used for the DNA extraction. qPCR was performed, using a Femto Bacterial DNA Quantification Kit (Zymoresearch, Inc., CA, USA) and an AB 7500 machine (Applied Biosystems, Inc., MA, USA).

\section{NGS-based $16 \mathrm{~S}$ gene analysis}


Extracted DNA from whole blood was amplified on the whole 16S coding region (V1-9) by PCR, using the Bacterial 16S rDNA PCR Kit (Takara Bio Inc., Shiga, Japan ) and MiniAmp Plus Thermal Cycler (Applied Biosystems). The amplicons were cleaned using NucleoSpin Gel and PCR Clean-up (MACHEREY-NAGEL $\mathrm{GmbH} \& \mathrm{Co} . \mathrm{KG}$ ). The purified amplicons were amplified again on the $16 \mathrm{~S}$ V3-V4 region, using Phusion High-Fidelity PCR Master Mix (New England Biolabs, Inc., MA, USA) with specific primers and barcodes. The same volume of loading buffer (containing SYBR green) was mixed with PCR products, and electrophoresis on a $2 \%$ agarose gel was performed. Samples with a bright main strip between 400450bp were chosen for further experiments. PCR products were purified using the Qiagen Gel Extraction Kit (Qiagen GmbH, Hilden, Germany). The libraries were generated using NEBNext UltraTM DNA Library Prep Kit (New England Biolabs), and sequenced on an Illumina NovaSeq6000 (Illumina, Inc., CA, USA). Paired-end reads were assigned to samples based on their unique barcodes and truncated by cutting off the barcode and primer sequences. Paired-end reads were merged using FLASH (V1.2.7). Quality filtering on the raw tags was performed under specific conditions to obtain high-quality clean tags according to QIIME (V1.7.0). The tags were compared with the reference database using the UCHIME algorithm to detect chimaera sequences, and chimaera sequences were removed. Analysis of Similarities (Anosim) was performed using QIIME software (Version 1.7.0). LEfSe analysis was conducted using the LEfSe software.

\section{Statistical analysis}

All statistical analyses were performed using JMP or EZR, except for the NGS analyses. Statistical tests performed for each dataset and significance levels are indicated in the figure legends.

\section{Results}

\section{Oral DSS administration triggers a peripheral SpA phenotype in SKG mice.}

To investigate whether oral DSS administration solely provokes SpA features in SKG mice under SPF conditions, female SKG mice and BALB/c mice aged 8- 12 weeks ( $n=10$ per group) were administered 1\% DSS in drinking water for 2 weeks under SPF conditions (Fig. 1a). Body weights, arthritis, and enthesitis scores were recorded every two weeks over 12 weeks. At week 12, there was no significant difference in the rate of body weight change between SKG mice and BALB/c mice (Fig. 1b). The incidence rates of arthritis and enthesitis at week 12 were $40 \%$ and 50\% in the SKG mice group, respectively (Fig. 1c,e). Arthritis and enthesitis composite scores in SKG mice increased chronologically, while none of those of BALB/c mice did (Fig. 1 d,f). In SKG mice, deviation and/or uplift of the plantar aponeurosis over the angle of the ankle were confirmed (Fig. 1g). H\&E staining revealed outstanding cell infiltration in the Achilles tendon and plantar aponeurosis in DSS-treated SKG mice. Slight cell infiltration adjacent to the vertebral disk was confirmed only in the DSS-treated SKG mice group (Fig. 1h).

\section{Oral DSS activates both Th1 and Th17 immunity in DSS-treated SKG mice.}


We then evaluated the changes in Th cell populations in the spleen, before and after DSS administration in SKG mice and BALB/c mice ( $n=5$ per group). Before DSS administration, SKG mice possessed a significantly higher percentage of both IFN- $\gamma$-producing Th cells (Th1 cells) and IL-17A-producing Th cells (Th17 cells) than BALB/c mice. After 2 weeks of DSS administration, the percentage of Th1 cells increased significantly in both SKG mice and BALB/C mice $(P<0.01)$, and that of Th17 cells was significantly increased in SKG mice $(P<0.05)$. (Fig. 2a-C). The median of increase fold of Th1 and Th17 cells in SKG mice was 1.73 and 1.48, respectively. These results suggest that oral DSS administration activates both systemic Th1 and Th17 immunity in SKG mice.

\section{Antibiotics, but not an anti-fungal agent, ameliorate DSS-induced peripheral SpA of SKG mice.}

To investigate which is pivotal, bacterial or fungal translocation in the pathogenesis of DSS-induced pSpA features in SKG mice, we administered antibiotics (MEPM plus VCM) or an anti-fungal agent (AMPH-B) in drinking water for 1 week from the time point 2 weeks before the initiation of DSS administration in another set of cohorts. In addition, $6 \%$ DMSO was added to the drinking water in the $1 \mathrm{st}$ week in each group to dissolve AMPH-B in drinking water. Female SKG mice aged of 8- 12 weeks $(n=10$ per group) were administered DMSO only, DMSO and antibiotics (MEPM and VCM), and DMSO and AMPH-B, respectively. After 1 week of free water drinking, $1 \%$ DSS in drinking water was administered for 2 weeks. All experiments were conducted under specific pathogen-free conditions. Body weights, arthritis, and enthesitis scores were recorded every two weeks from the initiation of DSS administration over 12 weeks (Fig. 3a). At week 12, there was no significant difference in the rate of change in body weight between SKG mice treated with DMSO only, DMSO plus MEPM and VCM, and DMSO plus AMPH-B (Fig. $3 b)$. There was no apparent difference in arthritis incidence rate or significant difference in arthritis scores between the three groups, which could be explained by the effect of DMSO (Fig. 3c,d). On the other hand, the incidence rate of enthesitis and enthesitis scores at week 12 was lower (zero) in the group with MEPM and VCM, compared to the control group and the group with AMPH-B (Fig. 3e,f). We also examined the difference in the percentage of Th cell populations in the spleen, between the group with MEPM and VCM and the control group after 2 weeks of DSS administration. As a result, the percentage of both Th1 and Th17 cells in the spleen was significantly lower in the group with MEPM and VCM compared than in the control group with DMSO only (Fig. 3g,h). These data indicate that bacterial but not fungal translocation plays an essential role in the pathogenesis of DSS-induced pSpA in SKG mice.

\section{Oral DSS increases not only amounts of bacterial DNA but also the number of species in the whole blood.}

DNA from whole blood was extracted in another set of cohorts to confirm that bacterial translocation was caused by DSS administration and to investigate its chronological changes in quantity and quality: SKG mice and BALB/c mice at day 0 , day 1 , day 7 , and day 14 ( $n=5$ per group). The amount of bacterial DNA was evaluated by qPCR on the 16S rRNA V3-V4 coding gene. In addition, NGS-based 16S gene analyses were performed to determine the profile of bacterial DNA in whole blood. By qPCR, it was clarified that SKG mice intrinsically tended to have more bacterial DNA in whole blood than BALB/c mice $(p=0.058)$, 
and the discrepancy became even larger and significant on day $1(P=0.034)$. The amount of circulating bacterial DNA in BALB/c mice elevated at day 7 with a delay compared to SKG mice (Fig. 4a). This chronological change, especially on days 0 and 1 might reflect the vulnerability of the gut in SKG mice due to dysbiosis caused by their genetic background. It was also revealed that the number of bacterial species commonly detected in individual five mice greatly increased after 2 weeks of DSS administration in both BALB/c mice and SKG mice: 80 to 393 , and 77 to 458 , respectively (Fig. 4b).

\section{Specific bacterial DNA are detected in the whole blood of DSS-treated SKG mice.}

To determine whether bacterial DNA specific to the DSS-treated SKG mice group was detected in whole blood, an Anosim test and a LEfSe analysis was performed with the same NGS dataset. An Anosim test was performed to examine whether there was a significant $\beta$ diversity between the group of SKG mice with DSS and the other three groups ( $n=5$ per group). It clarified that there was a significant $\beta$ diversity between the group of SKG mice with DSS and the group of BALB/c mice without DSS ( $r=0.6$ and $p=$ $0.016)$, the group of BALB/c mice with DSS ( $r=0.54$ and $p=0.01)$, and the group of SKG mice without DSS ( $r=0.82$ and $p=0.01)$, respectively (Table1). The LEfSe analysis was performed with the same NGS dataset to clarify the bacterial genera and species specific to the group of SKG mice with DSS, setting the significant p-value was $<0.05$, and the cut off of linear discriminant analysis (LDA) score was $4.0(\log 10)$; Lactobacillus reuteri, Sphingomonas, and Akkermansia were detected as significantly specific genera or species in the whole blood of the group of SKG mice with DSS (Fig.5).

\section{Discussion}

This study demonstrated that oral DSS administration in SKG mice under SPF conditions solely elicited pSpA features and a pathological axial feature and that this was associated with bacterial but not fungal translocation. Enthesitis was confirmed in at least $50 \%$ of the DSS-administered SKG mice. To be fair and to emphasise clinical importance, we adopted an original strict scoring system for enthesitis, so slight changes such as just widening of the ankle were not counted. Oral DSS administration solely provokes pSpA features in SKG mice, with bacterial dissemination into the blood. This oral DSS-induced pSpA features in SKG mice were suppressed by anti-bacterial agents (MEPM plus VCM), which are known as broad-spectrum antibiotics, but not by an anti-fungal agent (AMPH-B), which is also known as a broadspectrum anti-fungal agent. These results suggest that, bacterial factors are more important than fungal factors in the pathogenesis of oral DSS-induced pSpA. In fact, although there have been contradictory results, several reports have shown that antibiotics might be effective in reducing the disease severity of $\mathrm{SpA}$ [17-19]. Other reports have shown that antibiotics also have therapeutic potential for the treatment of IBDs $[20,21]$. In addition to peripheral enthesitis, axial lesions were seen adjacent to the vertebral disk at pathological level. However, the cell infiltration level was milder than that in curdlan-treated SKG mice [10]. We then examined whether antibiotics and/or an anti-fungal agent have therapeutic effects. In the set of cohorts regarding the antibiotics and anti-fungal agent, the incidence and score of enthesitis were suppressed by antibiotics (MEPM plus VCM), not an anti-fungal agent (AMPH-B). Arthritis scores unexpectedly tended to improve even in the control group with DMSO only, which might be explained by 
that DMSO is known for its therapeutic effects on arthritis and other inflammatory diseases [22-25]. DMSO did not influence the incidence rate of enthesitis and its score in the control group. This suggests that there are different mechanisms between DSS-induced enthesitis and arthritis, and further research is needed.

We also investigated changes in Th1 and Th17 cell levels in the spleen, because they are thought to represent changes in the systemic immune balance. It has been reported that intraperitoneal injection of $\beta$-glucan increases Th17 cells 3-4 folds in the lymph nodes of SKG mice, and that T cells sorted from normal SKG mice treated with $\beta$-glucan possess the potential to cause arthritis in recipient RAG2 KO mice, but not from IL-17A KO SKG mice. Therefore, Th17 cells are thought to play pivotal roles in the pathogenesis of arthritis in SKG mice [8]. In this context, it is noteworthy that in our studies, the percentage of Th1 cells increased by the same or more folds (the median was 1.73 and 1.48) than that of Th17 cells, and that Th1 cells increased with an even lower p-value than Th17 cells in our DSS-treated SKG mice. The increase in Th1 cell levels has been observed in IBD mouse models using wild-type mice with oral DSS administration [26,27]. While anti-IL-12/IL-23 antibody (ustekinumab) has been used to treat IBDs in clinical settings [28-31], anti-IL-17A antibodies are generally thought to be ineffective or contraindicated [32-34] . These findings suggest that SKG mice, which cause autoimmune and autoinflammatory phenomena due to $T$ cell signal dysfunction, can show a variety of phenotypes depending on additional environments and adjuvants. Many of autoimmune/autoinflammatory diseases and immune deficiency diseases have been known to be associated with $T$ cell signal dysfunction $[35,36]$. Our studies suggest that DSS administration in SKG mice with the Zap70 W163C mutation stimulates not only systemic Th17 immunity but also Th1 immunity via bacterial translocation in the gut.

We also demonstrated that SKG mice intrinsically tended to have more bacterial DNA in whole blood than $\mathrm{BALB} / \mathrm{c}$ mice. As of day 1 after the initiation of oral DSS administration, SKG mice contained even more bacterial DNA in whole blood compared to BALB/c mice. These findings suggest that the gut vulnerability of SKG mice, or "leaky gut" due to T cell signal dysfunction. On the other hand, there was no significant difference in the amount of bacterial DNA in whole blood between BALB/c and SKG mice at day14. As a hypothesis for these results, BALB/c mice have a healthier intestinal environment than SKG mice, and the leakage of bacteria after DSS administration occurs more slowly than in SKG mice. In addition, the number of bacterial species which were commonly detected in all five mice per group were almost the same after 14days of DSS administration between BALB/c mice and SKG mice. Therefore, whether there are bacterial genera and/or species specific to the whole blood of the group of SKG mice with DSS was of great interest.

Then, we performed an Anosim test and a LEfSe analysis. An Anosim test, which indicates $\beta$ diversity, revealed that the group of SKG mice with DSS had significantly unique bacterial species' DNA in their whole blood, compared to any other three groups: BALB/c mice before DSS administration, BALB/c mice after DSS administration, and SKG mice before DSS administration. Then, using a LEfSe analysis, DNAs of Lactobacillus reuteri, genus of Sphingomonas, and genus of Akkermansia were detected as significantly increased bacteria with a high LDA score $($ LDA $>4.0(\log 10))$ in the whole blood of the group 
of SKG mice with DSS administration. Lactobacillus reuteri is known to play a role as a type I interferon (IFN) inducer and has the potential to exacerbate autoimmune colitis and autoimmune diseases such as systemic lupus erythematosus (SLE), depending on the situation $[37,38]$. Sphingomonas produces sphingolipids instead of lipopolysaccharide (LPS) and is known to stimulate NKT cells $[39,40]$.

Akkermansia feeds on mucin, and although it is known for its protective functions against inflammation, some reports have implied its contribution to the pathogenesis of autoimmune diseases [41-43]. These bacteria might be key players in the pathogenesis and therapeutic targets in some patients with SpA, and further research is required.

Our study has some limitations: First, all the experiments were conducted in a single facility. It is known that commensal microbial diversity depends on each animal facility. Second, we have not completely excluded the effects of antibiotics other than as germicides, although we selected antibiotics which don't have strong anti-inflammatory effects.

\section{Conclusion}

We have demonstrated that oral DSS administration solely provokes $\mathrm{pSpA}$ features in SKG mice with specific bacterial translocation and systemic Th1 and Th17 activation. This was suppressed by antibiotics, and not by an anti-fungal agent. Our current studies provide additional findings with previous reports on SpA in SKG mice, showing that gut bacterial translocation can play a pivotal role in the pathogenesis of SpA, and will contribute to unravel the whole scheme of SpA pathogenesis.

\section{Abbreviations}

Abx, antibiotics; AMPH-B, amphotericin B; Anosim, Analysis of Similarities; AS, ankylosing spondylitis; ASCA, anti-Saccharomyces cerevisiae antibody; DMSO, dimethyl sulfoxide; DSS, dextran sulfate sodium; FCM, flow cytometry; H\&E, hematoxylin and eosin; IBD, inflammatory bowel disease; IFN, interferon; LDA, linear discriminant analysis; LEfSe, linear discriminant analysis effect size; LPS, lipopolysaccharide; MEPM, meropenem trihydrate; MSK, musculoskeletal; NGS, next-generation sequencing; PsA, psoriatic arthritis; pSpA, peripheral spondyloarthritis; qPCR, quantitative polymerase chain reaction; ReA, reactive arthritis; SLE, systemic lupus erythematosus; SpA, spondyloarthritis; SPF, specific pathogen free; VCM, vancomycin hydrochloride.

\section{Declarations}

\section{Ethics Approval and Consent to Participate}

All experiments were approved by the Kyoto University Animal Ethics Committee.

\section{Consent for Publication}

Not Applicable. 
Availability of data and materials

The datasets used and/or analysed during the current study are available from the corresponding author on reasonable request.

\section{Competing Interests}

The authors declare that they have no competing interests.

\section{Funding}

This study was supported by Grant-in-Aid for Scientific Research ([grant number: 16K09890 and 20K08773]) from the Ministry of Education, Culture, Sports, Science and Technology, Japan (M. H.).

\section{Authors' contributions}

YT has full access to all of the data in the study and takes responsibility for the integrity of the data and the accuracy of the data analysis. YT, MH, and AM contributed to the conception and design. YT was performed both in vivo and in vitro experiments. MK, YN, MS, HT, HO, KK, SA, RN, KM, KM, HY, and MT conceived the project. All authors read and approved the final manuscript.

\section{Acknowledgements}

We thank Shimon Sakaguchi (Osaka University, Osaka, Japan) and Keiji Hirota (Kyoto University, Kyoto, Japan) for supplying the mice and for helpful discussions.

\section{References}

1. Schett G, Lories RJ, D'Agostino MA, Elewaut D, Kirkham B, Soriano ER, et al. Enthesitis: from pathophysiology to treatment. Nat Rev Rheumatol. 2017:13;731-741.

2. Garg N, van den Bosch F, Deodhar A. The concept of spondyloarthritis: where are we now? Best Pract Res Clin Rheumatol. 2014;28:663-72.

3. Lories RJ, Mclnnes IB. Primed for inflammation: enthesis-resident T cells. Nat Med. 2012:18:1018-9.

4. Van Praet L, Van den Bosch FE, Jacques P, Carron P, Jans L, Colman R, and et al. Microscopic gut inflammation in axial spondyloarthritis: a multiparametric predictive model. Ann Rheum Dis. 2013;72:414-7.

5. Olofsson T, Lindqvist E, Mogard E, Andréasson K, Marsal J, Geijer M, et al. Elevated faecal calprotectin is linked to worse disease status in axial spondyloarthritis: results from the SPARTAKUS cohort. Rheumatology (Oxford). 2019;58:1176-1187. 
6. Gracey E, Vereecke L, McGovern D, Fröhling M, Schett G, Danese S, et al. Revisiting the gut-joint axis: links between gut inflammation and spondyloarthritis. Nat Rev Rheumatol. 2020;16:415-433.

7. Sakaguchi N, Takahashi T, Hata H, Nomura T, Tagami T, Yamazaki S, et al. Altered thymic T-cell selection due to a mutation of the ZAP-70 gene causes autoimmune arthritis in mice. Nature. 2003;426:454-60.

8. Hirota $\mathrm{K}$, Hashimoto $\mathrm{M}$, Yoshitomi H, Tanaka S, Nomura T, Yamaguchi T, et al. T cell self-reactivity forms a cytokine milieu for spontaneous development of IL-17+ Th cells that cause autoimmune arthritis. J Exp Med. 2007;204:41-7.

9. Yoshitomi H, Sakaguchi N, Kobayashi K, Brown GD, Tagami T, Sakihama T, et al. A role for fungal \{beta\}-glucans and their receptor Dectin-1 in the induction of autoimmune arthritis in genetically susceptible mice. J Exp Med. 2005;201:949-60.

10. Ruutu M, Thomas G, Steck R, Degli-Esposti MA, Zinkernagel MS, Alexander K, et al. $\beta$-glucan triggers spondylarthritis and Crohn's disease-like ileitis in SKG mice. Arthritis Rheumatol. 2012;64:2211-22.

11. Rehaume LM, Mondot S, Aguirre de Cárcer D, Velasco J, Benham H, Hasnain SZ, et al. ZAP-70 genotype disrupts the relationship between microbiota and host, leading to spondyloarthritis and ileitis in SKG mice. Arthritis Rheumatol. $2014 ; 66: 2780-92$.

12. Benham H, Rehaume LM, Hasnain SZ, Velasco J, Baillet AC, Ruutu M, et al. Interleukin-23 mediates the intestinal response to microbial $\beta-1,3-$ glucan and the development of spondyloarthritis pathology in SKG mice. Arthritis Rheumatol. 2014;66:1755-67.

13. Hoffman IE, Demetter P, Peeters M, De Vos M, Mielants H, Veys EM, et al. Anti-saccharomyces cerevisiae IgA antibodies are raised in ankylosing spondylitis and undifferentiated spondyloarthropathy. Ann Rheum Dis. 2003;62:455-9.

14. Andretta MA, Vieira TD, Nishiara R, Skare TL. Anti-Saccharomyces cerevisiae (ASCA) and antiendomysial antibodies in spondyloarthritis. Rheumatol Int. 2012;32:551-4.

15. Maillet J, Ottaviani S, Tubach F, Roy C, Nicaise-Rolland P, Palazzo E, et al. Anti-Saccharomyces cerevisiae antibodies (ASCA) in spondyloarthritis: Prevalence and associated phenotype. Joint Bone Spine. 2016;83:665-668.

16. Lubberts E. The IL-23-IL-17 axis in inflammatory arthritis. Nat Rev Rheumatol. 2015;11:415-29.

17. Barber CE, Kim J, Inman RD, Esdaile JM, James MT. Antibiotics for treatment of reactive arthritis: a systematic review and metaanalysis. J Rheumatol. 2013;40:916-28.

18. Ogrendik M. Treatment of ankylosing spondylitis with moxifloxacin. South Med J. 2007;100:366-70. 
19. Yang L, Liu B, Zheng J, Huang J, Zhao Q, Liu J, et al. Rifaximin Alters Intestinal Microbiota and Prevents Progression of Ankylosing Spondylitis in Mice. Front Cell Infect Microbiol. 2019;9:44.

20. Ledder O, Turner D. Antibiotics in IBD: Still a Role in the Biological Era? Inflamm Bowel Dis. 2018;24:1676-1688.

21. laniro G, Tilg H, Gasbarrini A. Antibiotics as deep modulators of gut microbiota: between good and evil. Gut. 2016;65:1906-1915.

22. Elisia I, Nakamura H, Lam V, Hofs E, Cederberg R, Cait J, et al. DMSO Represses Inflammatory Cytokine Production from Human Blood Cells and Reduces Autoimmune Arthritis. PLoS One. 2016;11:e0152538.

23. Hollebeeck S, Raas T, Piront N, Schneider YJ, Toussaint O, Larondelle Y, et al. Dimethyl sulfoxide (DMSO) attenuates the inflammatory response in the in vitro intestinal Caco-2 cell model.

Toxicol Lett. 2011;206:268-75.

24. Sari E, Bakar B, Dincel GC, Budak Yildiran FA. Effects of DMSO on a rabbit ear hypertrophic scar model: A controlled randomized experimental study. J Plast Reconstr Aesthet Surg. 2017;70:509-517.

25. de Abreu Costa L, Henrique Fernandes Ottoni M, Dos Santos MG, Meireles AB, Gomes de Almeida V, de Fátima Pereira W, et al. Dimethyl Sulfoxide (DMSO) Decreases Cell Proliferation and TNF-a, IFN- $y$, and IL-2 Cytokines Production in Cultures of Peripheral Blood Lymphocytes. Molecules. 2017;22:1789.

26. Alex P, Zachos NC, Nguyen T, Gonzales L, Chen TE, Conklin LS, et al. Distinct cytokine patterns identified from multiplex profiles of murine DSS and TNBS-induced colitis. Inflamm Bowel Dis. 2009;15:341-52.

27. Xiao HT, Peng J, Wen B, Hu DD, Hu XP, Shen XC, et al. Indigo Naturalis Suppresses Colonic Oxidative Stress and Th1/Th17 Responses of DSS-Induced Colitis in Mice. Oxid Med Cell Longev. 2019;2019:9480945.

28. Panaccione R, Danese S, Sandborn WJ, O'Brien CD, Zhou Y, Zhang H, et al. Ustekinumab is effective and safe for ulcerative colitis through 2 years of maintenance therapy. Aliment Pharmacol Ther. 2020;52:1658-1675.

29. Amiot A, Filippi J, Abitbol V, Cadiot G, Laharie D, Serrero M, et al. Effectiveness and safety of ustekinumab induction therapy for 103 patients with ulcerative colitis: a GETAID multicentre real-world cohort study. Aliment Pharmacol Ther. 2020;51:1039-1046.

30. Sands BE, Sandborn WJ, Panaccione R, O'Brien CD, Zhang H, Johanns J, et al. Ustekinumab as Induction and Maintenance Therapy for Ulcerative Colitis. N Engl J Med. 2019;381:1201-1214. 
31. Biemans VBC, van der Meulen-de Jong AE, van der Woude CJ, Löwenberg M, Dijkstra G, Oldenburg B, et al. Ustekinumab for Crohn's Disease: Results of the ICC Registry, a Nationwide Prospective Observational Cohort Study. J Crohns Colitis. 2020;14:33-45.

32. Hueber W, Sands BE, Lewitzky S, Vandemeulebroecke M, Reinisch W, Higgins PD, et al. Secukinumab, a human anti-IL-17A monoclonal antibody, for moderate to severe Crohn's disease: unexpected results of a randomised, double-blind placebo-controlled trial. Gut. 2012;61:1693-700.

33. Fauny M, Moulin D, D'Amico F, Netter P, Petitpain N, Arnone D, et al. Paradoxical gastrointestinal effects of interleukin-17 blockers. Ann Rheum Dis. 2020;79:1132-1138.

34. Rodríguez Moncada R, Vázquez Morón JM, Pallarés Manrique H. The onset of ulcerative colitis during treatment with secukinumab: can anti-IL-17A be a trigger for inflammatory bowel disease? Rev Esp Enferm Dig. 2019;111:720-721.

35. Au-Yeung BB, Shah NH, Shen L, Weiss A. ZAP-70 in Signaling, Biology, and Disease. Annu Rev Immunol. 2018;36:127-156.

36. Chan AY, Punwani D, Kadlecek TA, Cowan MJ, Olson JL, Mathes EF, et al. A novel human autoimmune syndrome caused by combined hypomorphic and activating mutations in ZAP-70. J Exp Med. 2016;213:155-65.

37. Fine RL, Mubiru DL, Kriegel MA. Friend or foe? Lactobacillus in the context of autoimmune disease. Adv Immunol. 2020;146:29-56.

38. Zegarra-Ruiz DF, El Beidaq A, Iñiguez AJ, Lubrano Di Ricco M, Manfredo Vieira S, Ruff WE, Mubiru D, et al. A Diet-Sensitive Commensal Lactobacillus Strain Mediates TLR7-Dependent Systemic Autoimmunity. Cell Host Microbe. 2019;25:113-127.e6.

39. Sriram V, Du W, Gervay-Hague J, Brutkiewicz RR. Cell wall glycosphingolipids of Sphingomonas paucimobilis are CD1d-specific ligands for NKT cells. Eur J Immunol. 2005;35:1692-701.

40. Kinjo Y, Pei B, Bufali S, Raju R, Richardson SK, Imamura M, Fujio M, et al. Natural Sphingomonas glycolipids vary greatly in their ability to activate natural killer T cells. Chem Biol. 2008;15:654-64.

41. Bian X, Wu W, Yang L, Lv L, Wang Q, Li Y, et al. Administration of Akkermansia muciniphila Ameliorates Dextran Sulfate Sodium-Induced Ulcerative Colitis in Mice. Front Microbiol. 2019;10:2259.

42. Berer K, Gerdes LA, Cekanaviciute E, Jia X, Xiao L, Xia Z, et al. Gut microbiota from multiple sclerosis patients enables spontaneous autoimmune encephalomyelitis in mice. Proc Natl Acad Sci U S A. 2017; 114:10719-10724.

43. Liu S, Rezende RM, Moreira TG, Tankou SK, Cox LM, Wu M, et al. Oral administration of miR-30d from Feces of MS Patients Suppresses MS-like Symptoms in Mice by Expanding Akkermansia muciniphila. 


\section{Tables}

\section{Table 1 Results of Anosym test ( $n=5$ per cohort)}

\begin{tabular}{|l|l|l|l|}
\hline & & R value & P value \\
\hline BALB/C & vS BALB/C+DSS & 0.092 & 0.231 \\
\hline BALB/C & vS SKG & -0.06 & 0.71 \\
\hline BALB/C & vS SKG+DSS & 0.6 & 0.016 \\
\hline BALB/C+DSS & vs SKG & 0.456 & 0.028 \\
\hline BALB/C+DSS & vS SKG+DSS & 0.54 & 0.01 \\
\hline SKG & vS SKG+DSS & 0.82 & 0.01
\end{tabular}

\section{Figures}

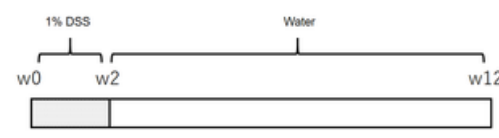

a

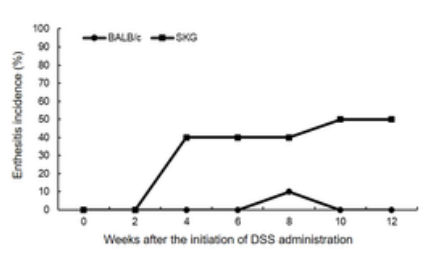

e

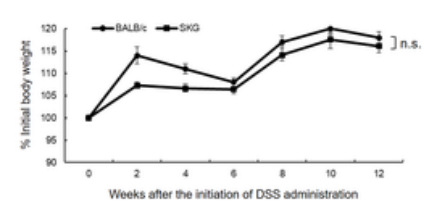

b

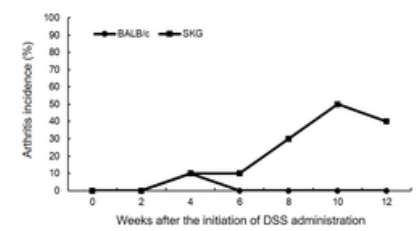

C

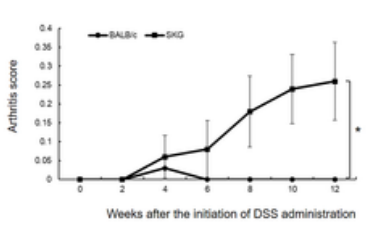

d

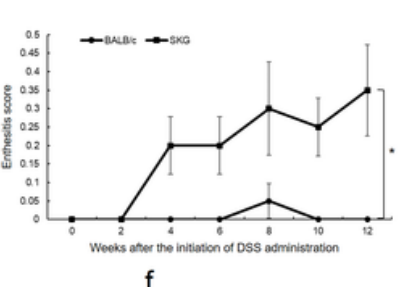

$f$

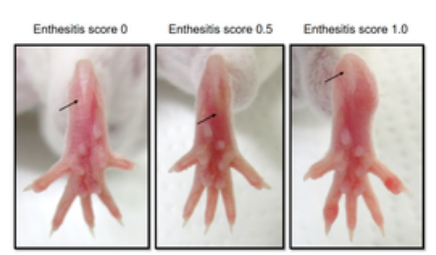

g

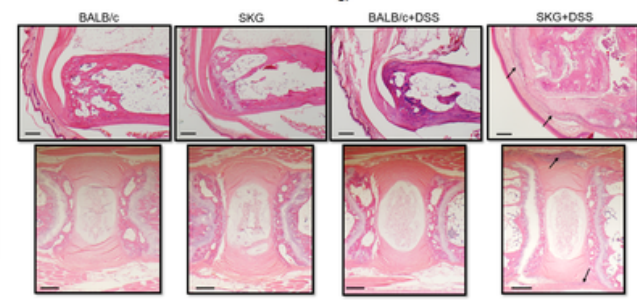

h

Figure 1

Oral DSS administration provokes SpA features in SKG mice. a Schematic schedule of mice treated with oral DSS. One percent of DSS was given in drinking water for 2 weeks, followed by regular water. Ten mice were used in each group. b There was no recorded decrease in body weight in either BALB/c or SKG mice. $c, d, e, f$ Arthritis incidence rate, arthritis score, enthesitis incidence rate, and enthesitis score were scored every 2 weeks. Values are expressed as mean \pm standard error of the mean (SEM). $g$ Representative photos of hind paws for the original enthesitis evaluation method . h Representative hematoxylin and eosin-stained sections of the peripheral enthesis (Achilles tendon) and spine (caudal vertebrae). Only DSS-treated SKG mice showed marked cell infiltration around the Achilles tendon and 
plantar aponeurosis (arrows), and slight cell infiltration around but not inside the annulus fibrosus of the intervertebral disk (arrows). Scale bar $=200 \mu \mathrm{m}$. Statistical analyses were performed by using the MannWhitney $U$ test. $(\mathbb{D}=\mathrm{P}<0.05$. $)$
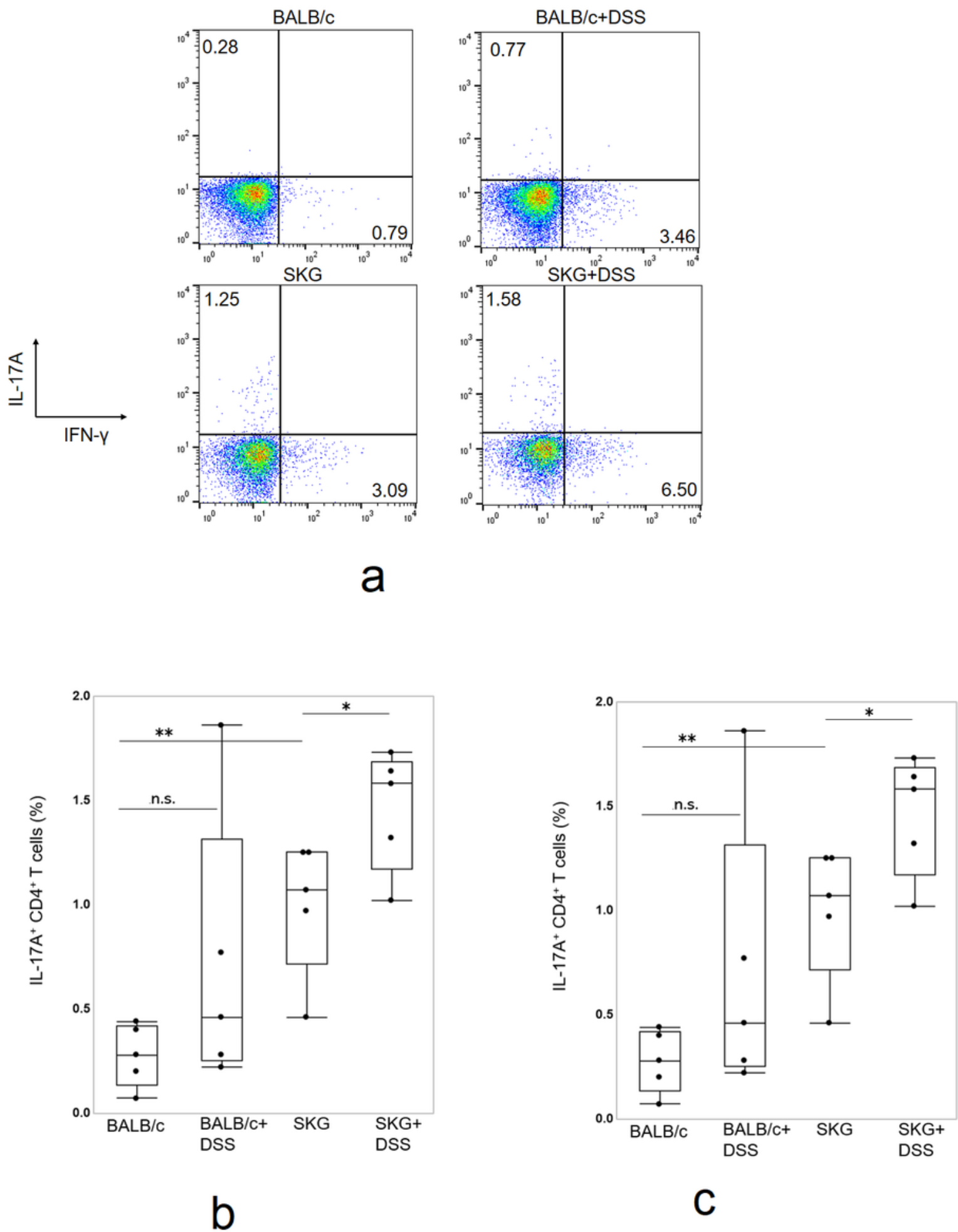

Figure 2

Oral DSS activates Th1 immunity and Th17 immunity in the spleen of DSS-treated SKG mice. a Representative FCM plots of splenic CD4+T cells at w0 and w12. b IFN-y positivity of splenic CD4+ T cells 
in BALB/c mice and SKG mice at w0 and w12. SKG mice originally possessed significantly more percentage of IFN- - -producing CD4+ T cells than BALB/c mice. DSS-treated SKG mice showed a significantly higher percentage of IFN- $y$-producing CD4+ T cells than untreated SKG mice. c IL-17Apositivity of splenic CD4+ T cells in BALB/c mice and SKG mice at w0 and w12. SKG mice originally possessed a significantly higher percentage of IL-17A-producing CD4+ T cells than BALB/c mice. DSStreated SKG mice showed a significant increase in the percentage of IL-17A-producing CD4+ T cells compared to untreated SKG mice. Statistical analyses were performed by using the Mann-Whitney $U$ test.

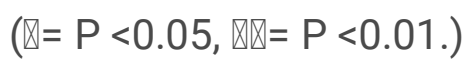

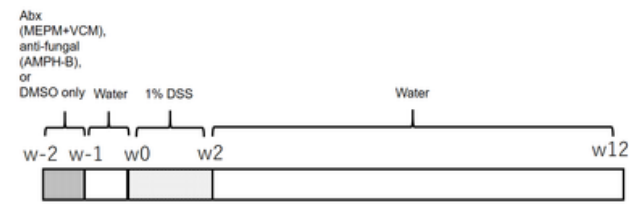

a

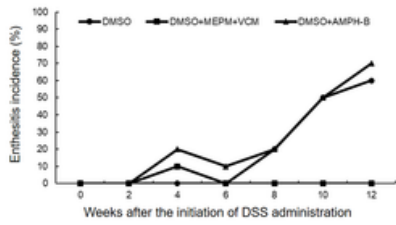

e

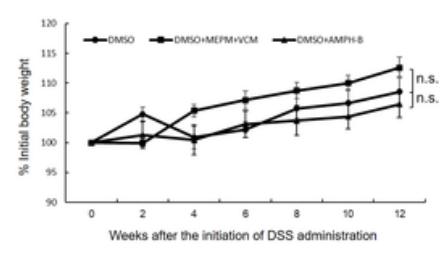

b

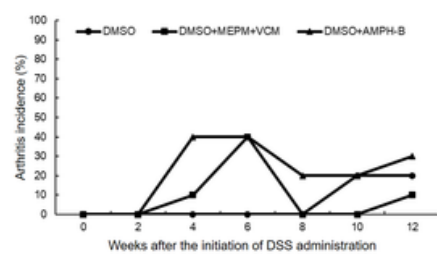

C

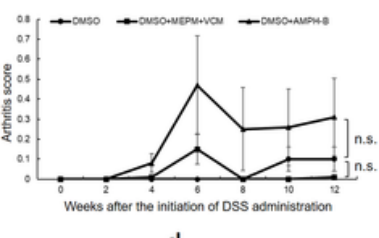

d

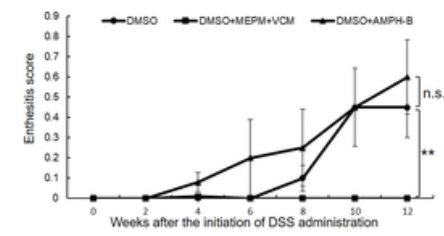

f
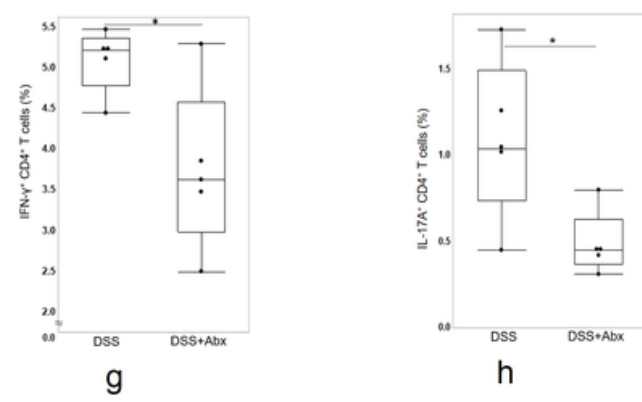

\section{Figure 3}

Antibiotics, but not an anti-fungal agent, ameliorate DSS-induced peripheral SpA of SKG mice. a Schematic schedule of mice treated with oral antibiotics (MEPM $(1 \mathrm{~g} / \mathrm{L})+\mathrm{VCM}(0.5 \mathrm{~g} / \mathrm{L}))$ or an anti-fungal agent (AMPH-B $(0.3 \mathrm{~g} / \mathrm{L}))$ and DSS. Antibiotics or an anti-fungal agent were administered in drinking water with $6 \%$ DMSO for 1 week, followed by regular water for 1 week. Then, $1 \%$ DSS was administered in drinking water for 2 weeks, followed by regular water. Ten mice were used in each group. Abx: antibiotics. b There was no recorded decrease in body weight in either BALB/c or SKG mice. c, d, e, f Arthritis incidence rate, arthritis score, enthesitis incidence rate, and enthesitis score were scored every 2 weeks. Values are the mean \pm standard error of the mean (SEM). Enthesitis was suppressed in the group treated with antibiotics (MEPM+VCM). $g$, h Results of FCM analysis in another set of cohorts. IFN-y positivity of splenic CD4+ T cells in SKG mice administered only DMSO and DMSO plus antibiotics (MEPM+VCM). Antibiotic-pretreated SKG mice showed a significantly lower percentage of IFN- $\gamma$-producing CD4+ T cells than control SKG mice after 2 weeks of DSS administration (g). Antibiotic-pretreated SKG mice also showed a significantly lower percentage of IL-17A-producing CD4+ T cells than control SKG mice after 2 weeks of DSS administration (h). Five mice per group were used. Statistical analyses were performed by

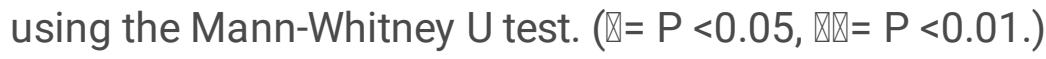




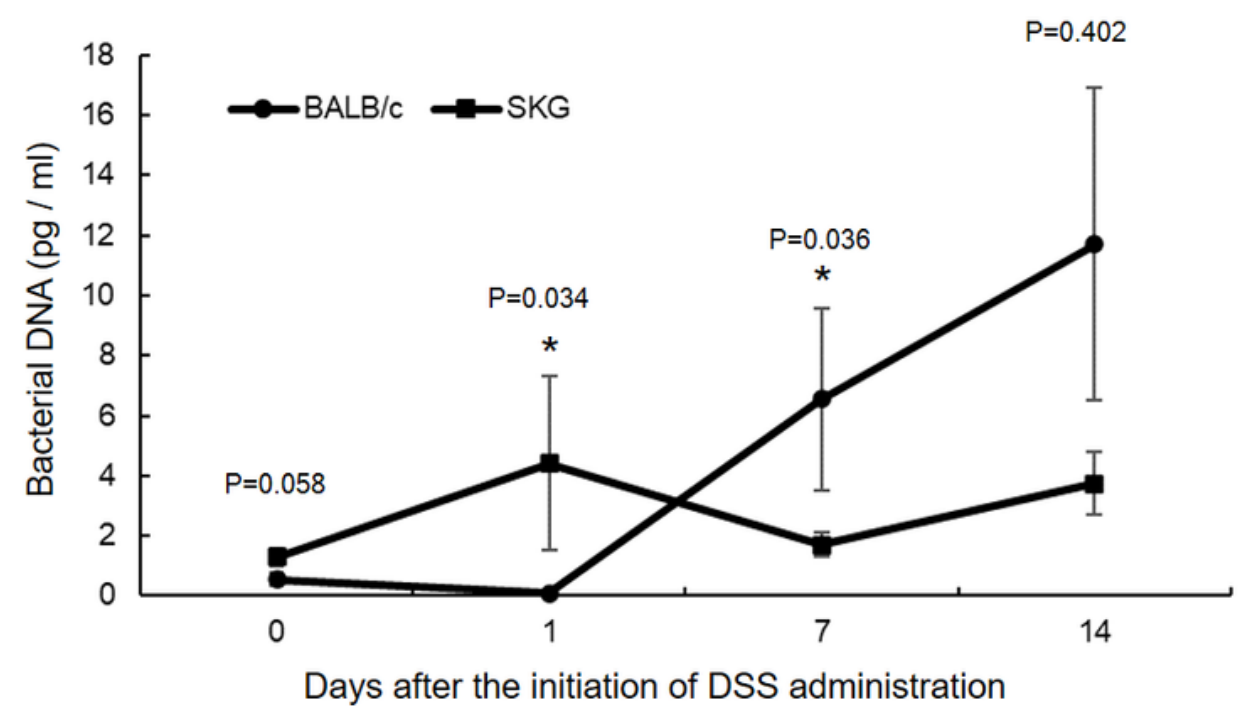

a
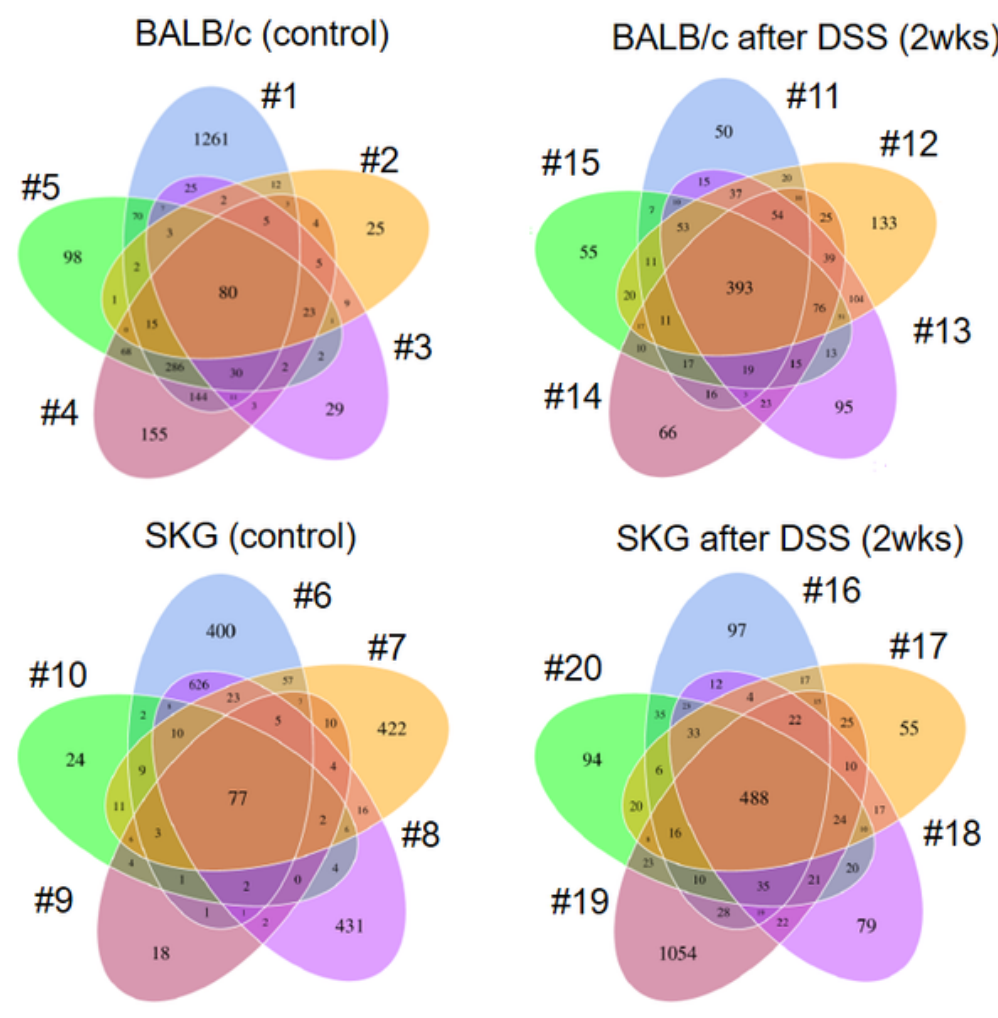

b

\section{Figure 4}

Oral DSS increases not only the amount of circulating bacterial DNA but also the number of species. a. The amount of bacterial DNA in the whole blood was measured by qPCR, $0,1,7$, and 14 days after oral DSS administration was initiated in BALB/C and SKG mice. SKG mice intrinsically tended to have more circulating bacterial DNA than BALB/c mice, and the discrepancy became even larger on day 1. In BALB/c mice, the amount of bacterial DNA was delayed compared to that in the SKG mice. Five mice per group 
were used. Values are presented as mean \pm SEM. Statistical analyses were performed using the MannWhitney $U$ test. $(\nabla=P<0.05) b$. Venn -diagrams, showing the number of bacterial species in circulating bacterial DNA at $\mathrm{d} 0$ and $\mathrm{d} 14$ after the initiation of DSS administration. Five mice were examined per group. The number of bacterial species commonly detected in all individual 5 mice per group increased in both in BALB/c and SKG mice at d 14. \# shows an identical number of mice. Twenty mice were examined as a whole.

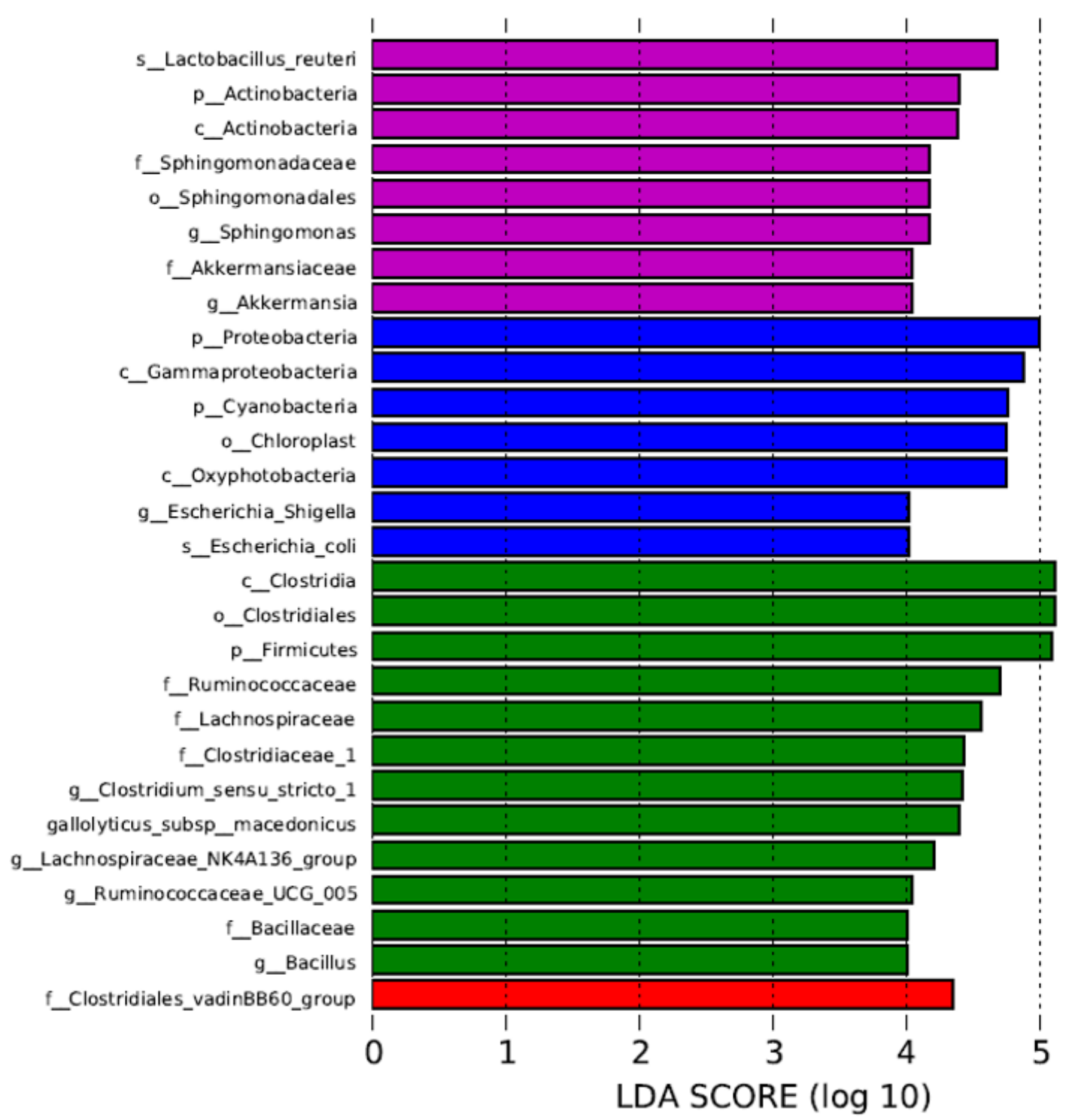

\section{SKG+DSS}

\section{SKG}

\section{$\mathrm{BALB} / \mathrm{c}+\mathrm{DSS}$}

\section{BALB/c} 6

\section{Figure 5}

Circulating DNA of significantly specific bacterial species are detected in the whole blood of DSS-treated SKG mice. a. Anosim test results for $\beta$ diversity at d0 (before DSS administration) and d 14 (after 2-week DSS administration). Significant $\beta$ diversities were confirmed between the group of DSS-treated SKG mice and the other three groups. Five mice per group were used. b. LEfSe analysis of circulating bacterial DNA in the whole blood at $d 14$. The cutoff was set as LDA score $>4.0$. Specific genera and species were detected in the DSS-treated SKG mice group. 